\title{
Study of the role played by NfsA, NfsB nitroreductase and NemA flavin reductase from Escherichia coli in the conversion of ethyl 2-(2'-nitrophenoxy)acetate to 4-hydroxy-(2H)-1,4-benzoxazin-3(4H)-one (D-DIBOA), a benzohydroxamic acid with interesting biological properties
}

\author{
Antonio Valle • Sylvie Le Borgne • Jorge Bolívar • \\ Gema Cabrera $\cdot$ Domingo Cantero
}

Received: 5 August 2011 /Revised: 6 November 2011 /Accepted: 23 November 2011 /Published online: 17 December 2011

(C) Springer-Verlag 2011

\begin{abstract}
Benzohydroxamic acids, such as 4-hydroxy-(2H)1,4-benzoxazin-3(4H)-one (D-DIBOA), exhibit interesting herbicidal, fungicidal and bactericidal properties. Recently, the chemical synthesis of D-DIBOA has been simplified to only two steps. In a previous paper, we demonstrated that the second step could be replaced by a biotransformation using Escherichia coli to reduce the nitro group of the precursor, ethyl 2-(2'-nitrophenoxy)acetate and obtain D-DIBOA. The $\mathrm{Nfs} A$ and $\mathrm{NfsB}$ nitroreductases and the NemA xenobiotic reductase of $E$. coli have the capacity to reduce one or two nitro groups from a wide variety of nitroaromatic compounds, which are similar to the precursor. By this reason, we hypothesised that these three enzymes could be involved in this
\end{abstract}

A. Valle, S. Le Borgne and J. Bolívar have contributed equally to this work.

A. Valle $(\bowtie) \cdot$ G. Cabrera $\cdot$ D. Cantero

Department of Chemical Engineering and Food Technology,

Campus de Excelencia Internacional Agroalimentario (ceiA3),

University of Cádiz,

Avda. República Saharaui s/n,

11510 Puerto Real, Cádiz, Spain

e-mail: antonio.valle@uca.es

S. Le Borgne

Department of Process and Technology,

Autonomous Metropolitan University-Cuajimalpa,

Artificios 40,

01120 México, DF, Mexico

J. Bolívar

Department of Biomedicine, Biotechnology and Public Health, Campus de Excelencia Internacional Agroalimentario (ceiA3),

University of Cádiz,

Avda República Saharui s/n,

11510 Puerto Real, Cádiz, Spain biotransformation. We have analysed the biotransformation yield (BY) of mutant strains in which one, two or three of these genes were knocked out, showing that only in the double $n f_{S} A / n f_{S} B$ and in the triple $n f_{S} A / n f_{S} B / n e m A$ mutants, the BY was $0 \%$. These results suggested that $\mathrm{Nfs} A$ and $\mathrm{NfsB}$ are responsible for the biotransformation in the tested conditions. To confirm this, the $n f s A$ and $n f s B$ open reading frames were cloned into the $\mathrm{pBAD}$ expression vector and transformed into the $n f_{S} A$ and $n f_{S} B$ single mutants, respectively. In both cases, the biotransformation capacity of the strains was recovered $(6.09 \pm 0.06 \%$ as in the wild-type strain) and incremented considerably when $\mathrm{NfsA}$ and $\mathrm{NfsB}$ were overexpressed $(40.33 \% \pm 9.42 \%$ and $59.68 \% \pm 2.0 \%$ respectively).

Keywords Biotransformation - Escherichia coli .

Benzohydroxamic acids · Nitroreductases NfsA and NfsB . Flavin reductase NemA $\cdot$ D-DIBOA

\section{Introduction}

Some benzohydroxamic acids isolated from plants such as 2,4-dihydroxy-(2H)-1,4-benzoxazin-3(4H)-one, known as DIBOA, and 2,4-dihydroxy-7-methoxy-(2H)-1,4-benzoxacin$3(4 \mathrm{H})$-one, known as DIMBOA (Honkanen and Virtanen 1960; Hamilton et al. 1962), exhibit a variety of interesting biological activities as phytotoxic properties, antimicrobial activity as well as antifeedant, antifungal and insecticidal properties (Duke 1986; Macías et al. 2006a, 2008, 2009).

Recently, the chemical synthesis of an analogue of these compounds known as D-DIBOA [4-hydroxy- $(2 \mathrm{H})-1,4$ benzoxazin-3(4H)-one] was simplified to only two steps 
(Macías et al. 2001, 2006b). The product of the first step is the ethyl 2-(2'-nitrophenoxy)acetate (Macías et al. 2006b) (Fig. 1a), which is henceforth referred to as the precursor (Valle et al. 2011). The second step involves the reduction of the nitro group followed by a cyclisation (Fig 1b). This second step, which occurs in complex conditions, requires the usage of an expensive catalyst and renders undesired products (Macías et al. 2006b). In a previous work, we demonstrated that this second step could be carried out by Escherichia coli JM109 (Fig. 1b) (Valle et al. 2011), and by this reason, we have approached microbial biotransformation as a new strategy of synthesis.

Aromatic nitroreductases have been widely studied in Gram-negative bacteria, mainly in the family Enterobacteriaceae. Phylogenetic analyses suggest that type I nitroreductases can be classified into two main groups, which are represented by the $E$. coli nitroreductases; NfsA group A (Blattner et al. 1997) and NfsB group B (Michael et al. 1994) encoded by the $n f_{s A}$ and $n f_{s} B$ genes, respectively. Group A nitroreductases are usually NADPH-dependent (Bryant et al. 1981; Zenno et al. 1996a), while group B nitroreductases may use both NADH or NADPH as electron donors (Bryant et al. 1981; Whiteway et al. 1998; Zenno et al. 1996b, 1998). In addition to the nitroreductases, bacteria also possess flavoreductases of the so-called old yellow enzyme (OYE) family that are capable of denitrating some explosives such as the nitroesters pentaerythritol tetranitrate (PETN), glycerol trinitrate (Roldán et al. 2008) and trinitrotoluene (TNT) (González-Pérez et al. 2007) using NADPH as the electron donor. The best known flavoreductases have been characterised in the species that belong to the family Enterobacteriaceae: the PETN reductase of Enterobacter cloacae (French et al. 1998), and the N-ethylmaleimide reductase xenobiotic (NemA) of E. coli encoded by the nemA gene (Williams et al. 2004). The physiological role of these enzymes has not been elucidated yet. Despite these flavoreductases have the same substrates range as the nitroreductases, they present significant differences in their amino acid sequences (Williams and Bruce 2002; Williams et al. 2004).

These three enzymes, NfsA, NfsB and NemA, which contain the flavin mononucleotide as prostetic group, can reduce up to two nitro groups $\left(-\mathrm{NO}_{2}{ }^{-}\right)$from different nitroaromatics compounds as TNT (Esteve-Nuñez et al. 2001;
Yin et al. 2005) to the corresponding amines $\left(-\mathrm{NH}_{2}\right)$ via hydroxylamino $(-\mathrm{NHOH})$ intermediates. The reduction is catalysed through the addition of one or two electrons from the cofactors NADH and/or NADPH (Roldán et al. 2008). This biotransformation is similar to the second step of the chemical synthesis of D-DIBOA from the precursor (Valle et al. 2011).

These antecedents motivated us to analyse whether the $n f_{S A} A, n f_{s} B$ and $n e m A$ gene products were involved in the conversion of the ethyl 2-(2'-nitrophenoxy)acetate to D-DIBOA.

\section{Materials and methods}

Bacterial strains, media and plasmids

E. coli strain $\mathrm{W} 3110\left[\mathrm{~F}^{-} \lambda^{-} r p h 1 \mathrm{INV}\right.$ rrnD-rrnE] was purchased from the American Type Culture Collection (ATCC 27325) and used as the wild-type strain in biotransformation and genes inactivation experiments. Strain AB502NemA is a derivative of $E$. coli $\mathrm{AB} 1157$ in which the three genes $n f s A, n f s B$ and $n e m A$ have been inactivated and was kindly donated by Dr. J.L. Ramos (Estación Experimental del Zaidín-CSIC, Granada, Spain; González-Pérez et al. 2007). E. coli DH5 $\alpha$ (supE44 $\Delta$ lacU169 $\$ 801 \mathrm{lacZ} \Delta \mathrm{M} 15$ hsdR17 recAl endA1 gyrA96 thi-l relA1) was used as the host for cloning the $n f_{S A}$ and $n f s B$ genes in the expression vector $\mathrm{pBAD} / \mathrm{His} \mathrm{A}$ (Invitrogen, Carlsbad, CA, USA). E. coli strains were cultured at $37^{\circ} \mathrm{C}$ in Luria-Bertani broth (LB) for molecular biology experiments and at $30^{\circ} \mathrm{C}$ for biotransformation experiments. When required, LB was supplemented with different antibiotics at the following final concentrations: ampicillin (Amp, $100 \mu \mathrm{g} / \mathrm{mL}$ ), kanamycin (Kan, $50 \mu \mathrm{g} / \mathrm{mL}$ ) or chloramphenicol $(\mathrm{Cm}, 25 \mu \mathrm{g} / \mathrm{mL})$. The pKD4, pKD46 and pCP20 plasmids were used for the inactivation of genes with PCR products as described by Datsenko and Wanner (2000). pKD46 is an easily curable, low copy number plasmid containing the lambda phage Red recombinase under the control of an arabinose inducible promoter containing the Cre recombinase (Datsenko and Wanner 2000). pKD4 is a template plasmid carrying a Kan resistance marker flanked by FLP recombinase recognition target sites. pCP20 is a plasmid conferring resistance to Amp and $\mathrm{Cm}$ that shows

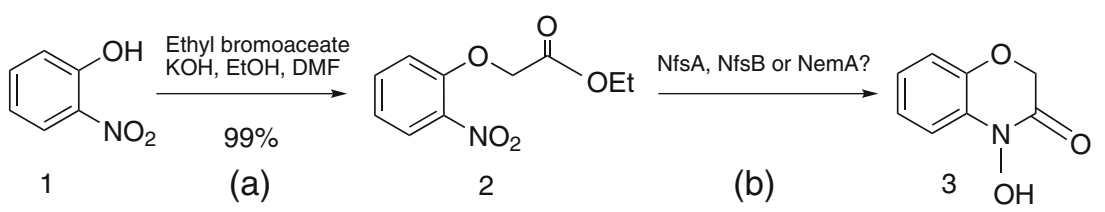

Fig. 1 Benzohydroxamic acid synthesis: a first step: synthesis of ethyl 2-(2'-nitrophenoxy)acetate (precursor) (2) using 2-nitrophenol (1) as starting material described by Macías et al. (2006b); b second step: hypothesis tested in this work of the precursor reduction by biotransformation with the NfsA, NfsB and/or NemA from $E$. coli 
temperature-sensitive replication and thermal induction of FLP recombinase synthesis. The $\mathrm{pBAD} / \mathrm{His} \mathrm{A}$ vector containing the $\operatorname{araC}$ promoter, which is inducible by arabinose was used in expression experiments.

Construction of the single mutants, $n f_{s} A, n f_{s} B$ and nem $A$, and double mutants, $n f_{S} A / n e m A, n f_{S} B / n e m A$ and $n f_{S} A / n f_{S} B$

The single and double mutants with $n f_{s} A, n f_{S} B$ and nem $A$ genes inactivated were obtained from PCR products of these genes disrupted with a Kan resistance marker following the method of Datsenko and Wanner (2000). For this purpose, pairs of primers (Table 1) were designed in order to have short 5' (H1) or 3' (H2) homology sequences of the target genes (in capital letters) that were flanking $\mathrm{P} 1$ or $\mathrm{P} 2$ priming sequences of the pKD4 vector (in small letters). Using these primers and the $\mathrm{pKD} 4$ vector as the template, PCR products containing the $\mathrm{Kan}^{\mathrm{R}}$ gene, flanked by FLP recognition targets (FRTs) were obtained. The PCR-generated products were introduced by electroporation into E. coli W3110 previously transformed with the pKD46 plasmid. The transformants were selected in LB agar plates supplemented with Kan and Amp. Gene disruptions were confirmed by PCR amplification using the following primers described in Table 1. The Kan resistance markers were removed after transformation with the pCP20 plasmid. Clones were selected by replica plating in LB agar plates supplemented with $\mathrm{Cm}$ or Kan. For plasmid curing, several clones were randomly selected, and replica was plated in LB agar plates with no antibiotic and incubated at $42^{\circ} \mathrm{C}$. The double mutants were generated from the single mutants using the same procedure described above.

\section{Cloning of the $n f_{S A}$ and $n f s B$ genes}

The $n f s A$ open reading frame (ORF) was PCR-amplified from $E$. coli W3110 chromosomal DNA using the primers in Table 1 containing NcoI or HindIII sites. PCR was performed using the i-pfU ${ }^{\mathrm{TM}}$ DNA polymerase (Intron, Gyeonggi-do, Korea) following the manufacturer's instructions. The PCR products were then digested with NcoI and HindIII (Takara, Shiga, Japan) and ligated into the pBAD/ His A vector digested with the same restriction enzymes. The resulting plasmids were named pBAD-NfsA and pBAD-NfsB. Approximately $25 \mathrm{ng}$ of each plasmid were transformed into the $n f_{S A} A$ and $n f_{s} B$ single mutants of $E$. coli $\mathrm{W} 3110$, respectively, to complement the inactivated genes. The resulting strains were named $n f s A \mathrm{pBAD}-\mathrm{NfsA}$ and $n f s B \mathrm{pBAD}-\mathrm{NfsB}$. In both cases, the cloned DNAs were sequenced with 3730XL DNA sequencer (Macrogen Inc, Seoul, Korea) and compared with the $n f_{s} A$ and $n f_{S} B$ sequences using the ClustalW software. The single mutants transformed

Table 1 Names and sequences of the primers designed for this study

\begin{tabular}{|c|c|}
\hline Primers & Sequences \\
\hline $\mathrm{H} 1 \mathrm{P} 1-n f_{S A}$ & CGC TGA CCG GCG GGC AAA AAC ACG TAG CGC AAG CGG gtg tag get gga get get tc \\
\hline $\mathrm{H} 2 \mathrm{P} 2-n f s A$ & AGA TCC GGA TTA TCC GCA GGC CAG CCA AGG CAC AGC cat atg aat atc ctc ctt a \\
\hline $\mathrm{H} 1 \mathrm{P} 1-n f_{s} B$ & ATG GAT ATC ATT TCT GTC GCC TTA AAG CGT CAT TCC gtg tag get gga get get tc \\
\hline $\mathrm{H} 2 \mathrm{P} 2-n f_{s} B$ & CCA TCC ACT CTG CAT CAT CAT GCA GAT CTT TAC GGT GCc ata tga ata tce tec tta \\
\hline H1P1-nemA & TAT TAG TGA AGC CAC GCA AAT TTC TGC CCA GGC AAA gtg tag get gga get get tc \\
\hline H2P2-nemA & CAG CGT TTC AGC TTT TTC TAC TGT GTA TGC ACC TGC cat atg aat atc ctc ctt a \\
\hline$n f s A-\mathrm{F}$ & GCG CGA TCG GTA AAT TGC CTA AAG \\
\hline$n f s A-\mathrm{R}$ & CAC GCA GCC GCT TAC ACG AAT AG \\
\hline$n f s B-F$ & TCA GTG TAT CCC GGC GAA GAA ATC \\
\hline$n f s B-\mathrm{R}$ & CAC GTT TTT CGA AGG TCC GCA G \\
\hline nemA-F & GCT GGA AAA TGG CCG TGA GAA CC \\
\hline nemA-R & CCA CGC CCC AGT TGT AGG TCA GTT C \\
\hline$N c o \mathrm{I}-n f s A-\mathrm{F}$ & 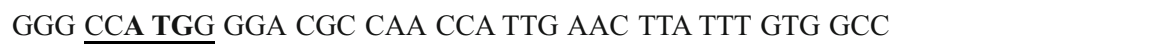 \\
\hline HindIII- $n f_{S} A-\mathrm{R}$ & 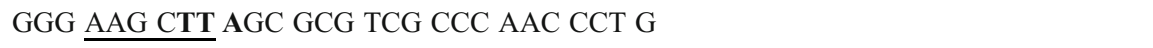 \\
\hline$N c o \mathrm{I}-n f s B-\mathrm{F}$ & 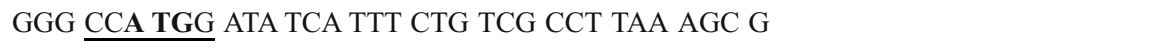 \\
\hline 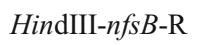 & 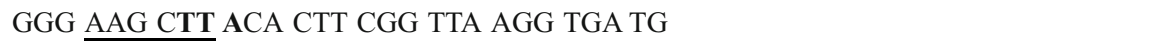 \\
\hline
\end{tabular}

The primers from 1 to 6 were used to obtain the single and double mutants of the $n f s A, n f s B$ and nemA genes. Capital letters indicate the homologous sequences of the target genes, and the lower case letters indicate the priming sequences of the pKD4 vector. In the names of the primers, H1P1 indicates the forward primers and H2P2 the reverse primers. Primers from 7 to 12 were used for PCR verification of the deletion of the targeted genes. The primers from 13 to 16 were designed for the cloning of the $n f s A$ and $n f s B$ ORFs into the pBAD vector. The start and stop codons are shown in bold letters. These primers incorporate restriction enzyme targets at $5^{\prime}$ end (underlined in the sequences). The restriction enzymes used are indicated in the name of each primer. Forward (-F) and reverse (-R) primers are also indicated 
with $\mathrm{pBAD} / \mathrm{His} \mathrm{A}$ empty vector were used as control and named $n f_{s} A \mathrm{pBAD}$ and $n f_{s} B \mathrm{pBAD}$.

The vector $\mathrm{pBAD}-\mathrm{Nfs} A$ construct was introduced into the $n f s B$ single mutant and into the wild-type strain (named $n f s B$ pBAD-NfsA and wtpBAD-NfsA respectively). The $n f s A$ single mutant and the wild-type strain were also transformed with the pBAD-NfsB plasmid (named $n f s A$ pBAD$\mathrm{NfsB}$ and wtpBAD-NfsB.

\section{Biotransformation experiments}

The inocula were prepared in $10 \mathrm{~mL}$ of $\mathrm{LB}$ medium and grown overnight. The cultures were then inoculated into $90 \mathrm{~mL}$ of LB medium in 250-mL Erlenmeyer flasks and supplemented with $1 \mathrm{~mL}$ of stock solution of precursor diluted in methanol $(\mathrm{MeOH})$ to achieve a final concentration of $2.22 \mathrm{mM}(0.5 \mathrm{mg} / \mathrm{mL})$ and $1 \%(v / v)$ of $\mathrm{MeOH}$, respectively. Cells were grown at $30^{\circ} \mathrm{C}$ and $150 \mathrm{rpm}$ for up to $26 \mathrm{~h}$. For biotransformation experiments in which the expression of NfsA or NfsB were controlled using the pBAD A vector, the LB medium was supplemented with Amp, and the cells were grown to an optical density $\left(\mathrm{OD}_{600 \mathrm{~nm}}\right)$ of 0.6 , and then the expression proteins were induced by addition of L-arabinose to a final concentration of $0.02,0.002$ or $0.0002 \%(w / v)$.

\section{Analytical techniques}

For quantitative analysis of the precursor and D-DIBOA, $1 \mathrm{~mL}$ samples were withdrawn from the cultures, centrifuged at $10,000 \times g$ for $10 \mathrm{~min}$ and filtered through $0.22-\mu \mathrm{m}$ nylon filters (VWR International Eurolab S.L., Barcelona, Spain) before analysis by reverse-phase high performance liquid chromatography (HPLC) on a Merck HITACHI HPLC system using a Phenomenex ${ }^{\circledR}$ Gemini C18 $4.6 \times 250 \mathrm{~mm}$ column as previously described by Valle et al. (2011). The biotransformation yield (BY) was calculated as follows:

$\mathrm{BY}=\frac{\text { molD }- \text { DIBOA }}{\text { molprecursor }} \times 100$

The cell dry weight (CDW) was quantified using standard procedures (Greenberg et al. 1992) in order to calculate the specific productivity (SP):

$\mathrm{SP}=\frac{\operatorname{mg}(\mathrm{D}-\mathrm{DIBOA})}{\operatorname{mg}(\mathrm{CDW}) \times \mathrm{h}}\left(\mathrm{h}^{-1}\right)$

The mutants and the wild-type BY and SP average data and standard deviation (SD) were calculated at least from three replicates. StatGraphics centurion for Windows version 16.0 was used for statistical analysis. The results were considered significantly different at $\alpha$ two-tailed level of 0.05 using the
Student's $t$ statistic function. Previously, the data were performed with Kolmogorov-Smirnov test for normality.

The GenBank/EMBL accession numbers for $n f_{S} A$ and $n f_{S} B$ ORF are EG11261 and EG20151, respectively.

\section{Results}

D-DIBOA in vivo synthesis by E. coli $\mathrm{W} 3110$

The biotransformation ability of the E. coli wild-type strain W3110 was evaluated in shake flasks cultures in which the LB medium was supplemented with the precursor by monitoring the bacterial growth, D-DIBOA and precursor concentrations at several times (Fig. 2). The analysis of the data obtained in these experiments showed that the precursor completely disappeared from the medium after $15 \mathrm{~h}$ of culture, but the BY remain virtually unchanged from 15 to $26 \mathrm{~h}$ (around 5.5\%). Therefore, the production of D-DIBOA could be evaluated at any moment during this interval of time. The disappearance of the precursor was due in part to the biotransformation to D-DIBOA; however, there was also a spontaneous degradation. This effect was previously observed in precursor's stability experiments in different aqueous solution (data not shown). This phenomenon can be observed in Fig. 2, in which the BY of D-DIBOA is 5.5\%; however, the precursor that remains after $15 \mathrm{~h}$ is not around $95 \%$ as can be expected, but the precursor is transformed completely in the medium. Despite the yield of the biological transformation is significantly lower than the chemical synthesis $(5.5 \%$ versus $77 \%$ respectively), these experiments are necessary to establish the wild-type biotransformation yield as a reference and therefore to be able to elucidate the role of $n f_{S} A, n f_{S} B$ and nemA genes in the biotransformation of the precursor to DDIBOA by knocking out these genes.

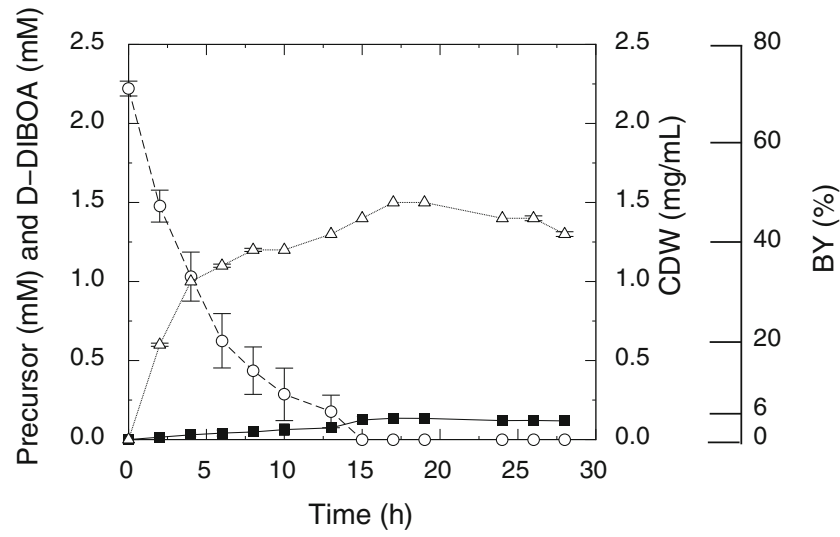

Fig. 2 Wild-type growth in the presence of precursor $(--\infty--)$ and production of D-DIBOA (- $\longrightarrow$ ). Concentrations are expressed in millimolars; CDW (................. is expressed in milligram per milliliter. The mean and SD values were calculated at least in three replicates 
Effect of the inactivation of the $n f_{S} A, n f_{S} B$ and $n e m A$ genes on the synthesis of D-DIBOA

To study the possible role of the E. coli nitroreductases $\mathrm{NfsA}$ and $\mathrm{NfsB}$ nitroreductases and NemA flavin reductase in the synthesis of D-DIBOA, we constructed $n f_{S} A, n f s B$ and $n e m A$ single mutants and $n f_{S} A / n f_{S} B, n f_{S} A / n e m A$ and $n f_{s} B /$ nem $A$ double mutants with these genes inactivated in $E$. coli W3110. The mutants and the wild-type strains were then grown for $26 \mathrm{~h}$ to ensure that the precursor had completely disappeared from the culture medium.

The analysis of these experiments showed that $n f_{S} A$ and $n f S B$ but not nemA single mutants had significant lower BY than the wild-type strain (Fig. 3). In this sense, the BY for $n f s A$ single mutant was $2.2 \pm 0.13 \%$ and $3.49 \pm 0.42 \%$ for $n f s B$ single mutant, which represent a decrease of biotransformation capacity of $59.7 \pm 2.38 \%$ and $35.92 \pm 7.64 \%$, respectively, compared to that of the wild-type strain $(5.45 \pm 0.56 \%)$. These results suggested that these two nitroreductases are involved in the synthesis of D-DIBOA. According to the BY presented in Fig. 3, NfsA has a more active role than NfsB in the in vivo synthesis of D-DIBOA. On the other hand, the nemA single mutant BY $(5.27 \pm 0.57 \%)$ was no significantly different from the wild-type, retaining a $96.70 \pm 10.42 \%$ of the biotransformation ability of the wild-type strain, indicating that the flavin reductase NemA is not involved in the synthesis of D-DIBOA.

We also carried out the same analysis for the double mutants mentioned above and we found, as expected, that the BY of the double mutants $n f_{S} A / n e m A$ and $n f_{S} B /$ nem $A$ were lower than that of the wild-type strain, with values of $2.75 \pm 0.18 \%$ and $3.81 \pm 0.63 \%$, respectively, representing a $49.49 \pm 3.30 \%$ and $29.99 \pm 11.50 \%$ decrease compared to the wild-type (Fig. 3). On the other hand, the BY of the $n f s B / n e m A$ double mutant did not present significant differences compared to the $n f s B$ single mutant (3.49\% $\pm 0.42 \%)$. However, unexpectedly, the BY of the double mutant $n f s A / n e m A$ was slight but significantly higher than for the $n f s A$ single mutant $(2.20 \% \pm 0.13 \%)$.
The $n f_{S} A / n f_{S} B$ double mutant and the $n f_{S} A / n f_{S} B / n e m A$ triple mutant (strain AB502NemA kindly provided by Dr. J.L. Ramos), were unable to synthesise D-DIBOA from the precursor and the BY and the SP were therefore $0 \%$ (Fig. 3). These results indicated that the NfsA and NfsB nitroreductases are the sole enzymes involved in the in vivo synthesis of D-DIBOA in our experimental conditions.

The SP observed in all mutants and wild-type, presented the same pattern as the BY, indicating that the bacterial growth did not affect D-DIBOA synthesis (Fig. 3).

Recovery of the biotransformation capacity of $n f s A$ and $n f s B$ single mutants

To further confirm the role of NfsA and NfsB in the synthesis of D-DIBOA, the $n f s A$ and $n f s B$ ORFs were cloned into the $\mathrm{pBAD} / \mathrm{His} \mathrm{A}$ expression vector and reintroduced into the single mutants in order to evaluate the complementation of the lost activities. In both cases, the BY at $16 \mathrm{~h}$ were calculated as the average of three different experiments using three clones, named $\mathrm{c} 1, \mathrm{c} 2$, and $\mathrm{c} 3$, selected from the same transformation (Fig. 4).

The BY obtained indicated that $n f_{s} A \mathrm{pBAD}-\mathrm{Nfs} A$ (Fig. 4a) and $n f_{s} B$ pBAD-NfsB (Fig. 4b) recovered their capacity to synthesise D-DIBOA from the precursor when the expression of both proteins were induced with $0.0002 \%$ $(w / v)$ of L-arabinose (BY of $5.69 \% \pm 0.60 \%$ and $5.44 \% \pm$ $1.14 \%$, respectively, versus $6.23 \% \pm 1.74 \%$ for the wildtype strain). Furthermore, the biotransformation capability of both strains was considerably increased when protein expression was induced at higher L-arabinose concentrations, reaching a maximum BY value of $40.33 \% \pm 9.42 \%$ in the case of NfsA (Fig. $4 \mathrm{a}$ ) and $59.68 \% \pm 2.0 \%$ in the NfsB (Fig. $4 \mathrm{~b})$ with $0.02 \%(w / v)$ of L-arabinose. These yields represented 6.5- and 9.6-fold increments in the BY for $n f_{s} A \mathrm{pBAD}-\mathrm{NfsA}$ and for $n f s B \mathrm{pBAD}-\mathrm{NfsB}$, respectively, compared to the wild-type, reaching yields of a similar range to the chemical synthesis $(77 \%)$. These increments were not

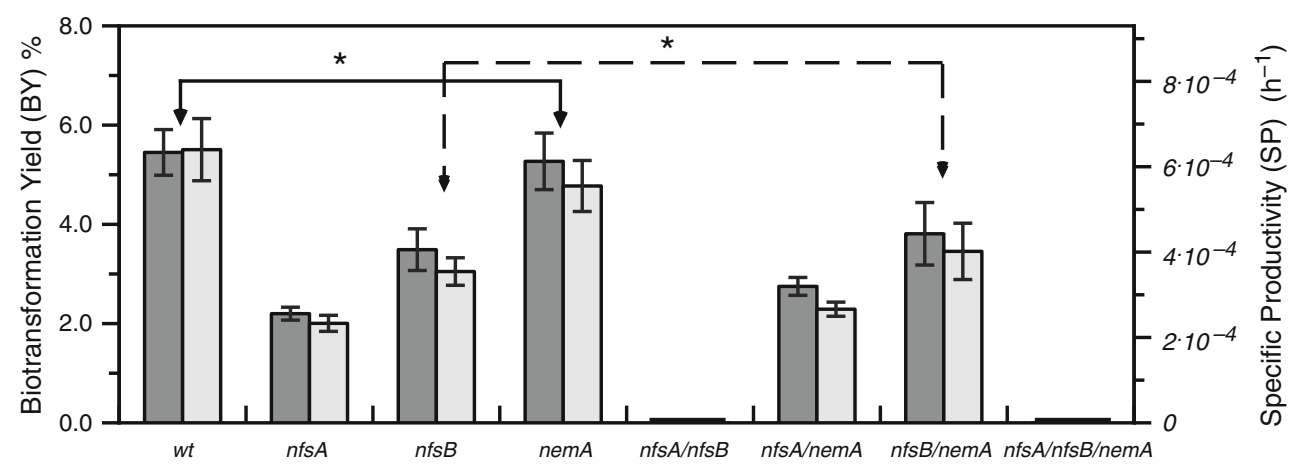

Fig. 3 Histograms of biotransformation yield (BY) coloured in dark grey and the specific productivity (SP) coloured in clear grey of singles and doubles mutants and the triple mutant at $26 \mathrm{~h}$ post inoculum. The $n f_{S A} A f_{s} B$ double mutant and the AB502NemA triple mutant were $0 \%$, for both parameters. Asterisks show the pair of strains that have not any significant differences $(P>0.05)$ nor BY neither RP 

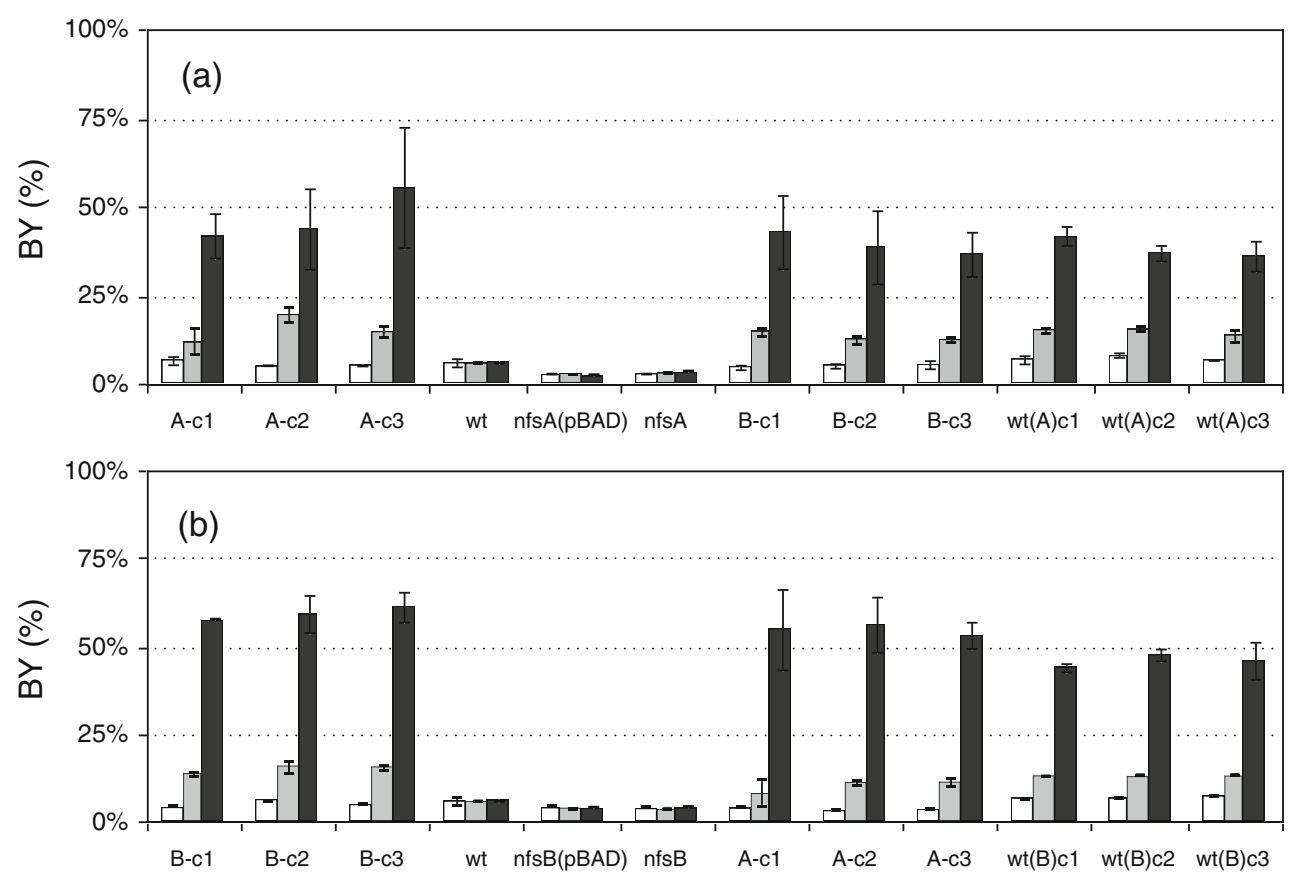

Fig. 4 Histograms of BY of three clones from the same transformant selected in LB-ampicillin. The three clones were named c1, c2 and c3. Prefix A indicate $n f s A$ single mutant and prefix B indicate $n f s B$ singles mutants. a Overexpression of NfsA protein in three clones of the mutants and wild-type strains; $n f s A \mathrm{pBAD}-\mathrm{Nfs} A$ (A-c1, A-c2 and Ac-3), $n f_{s} B \mathrm{pBAD}-\mathrm{NfsA}$ (B-c1, B-c2 and B-c3), wtpBAD-NfsA and the corresponding positive control of $n f s A$ mutant and the negative control $n f s A \mathrm{pBAD}$ and $\mathbf{b}$ overexpression of NfsB protein in three clones of the mutants and wild-type strains; $n f s B \mathrm{pBAD}-\mathrm{NfsB}$ (B-c1, B-c2 and B-c3), $n f s A \mathrm{pBAD}-\mathrm{NfsB}$ (A-c1, A-c2 and Ac-3) and wtpBAD-NfsB with their corresponding positive control of $n f s B$ mutant and the negative control $n f s B \mathrm{pBAD}$. Both histograms show in white bars the assays tested with $0.0002 \%$, in grey with $0.002 \%$ and in black $0.02 \%$ of L-arabinose. The error bars represent the SD of at least three independent replicates due neither to the presence of the expression vector nor to the inductor since the BY of the single mutants $n f_{S} A$ and $n f_{S} B$ transformed with the $\mathrm{pBAD} / \mathrm{His} \mathrm{A}$ empty vector and using the same L-arabinose concentrations were very similar to the BY of the non transformed mutants (Fig. 4).

In order to explore the effect caused (yields of conversion observed) by an extra copy of NfsA or NfsB or both genes, the $n f s B$ single mutant and the wild-type strain were transformed with pBAD-NfsA plasmid (named $n f s B \mathrm{pBAD}-\mathrm{NfsA}$ and wtpBAD-NfsA, respectively); on the other hand, the $n f s A$ single mutant and the wild-type strain were transformed with pBAD-NfsB construct (named $n f s A$ pBAD$\mathrm{NfsB}$ and wtpBAD-NfsB, respectively). The biotransformation ability of these strains was analysed in the same experimental conditions previously described. We found that there was no increment of the BY when the $n f s A \mathrm{pBAD}$ $\mathrm{NfsA}$ strain was compared with $n f_{s} B(\mathrm{pBAD}-\mathrm{Nfs} \mathrm{A})$ and wtpBAD-NfsA strains (Fig. 4a). A similar behaviour regarding the BY was observed when the $n f_{s} B \mathrm{pBAD}-\mathrm{NfsB}$ strain was compared with $n f s A \mathrm{pBAD}-\mathrm{NfsB}$ and wtpBAD-NfsB strains (Fig. 4b).

All the assays previously described were carried out $16 \mathrm{~h}$ after inoculation because in the wild-type strain the maximum BY was reached at this time. However, in order to evaluate how the biotransformation capacity of these strains varies in time, clones c2 of $n f s A \mathrm{pBAD}-\mathrm{Nfs} \mathrm{A}$ and $\mathrm{c} 3$ of $n f s B \mathrm{pBAD}-$ $\mathrm{NfsB}$ were supplemented with $0.02 \%$ of $\mathrm{L}$-arabinose and the BY for both clones was monitored at several times (Fig. 5).

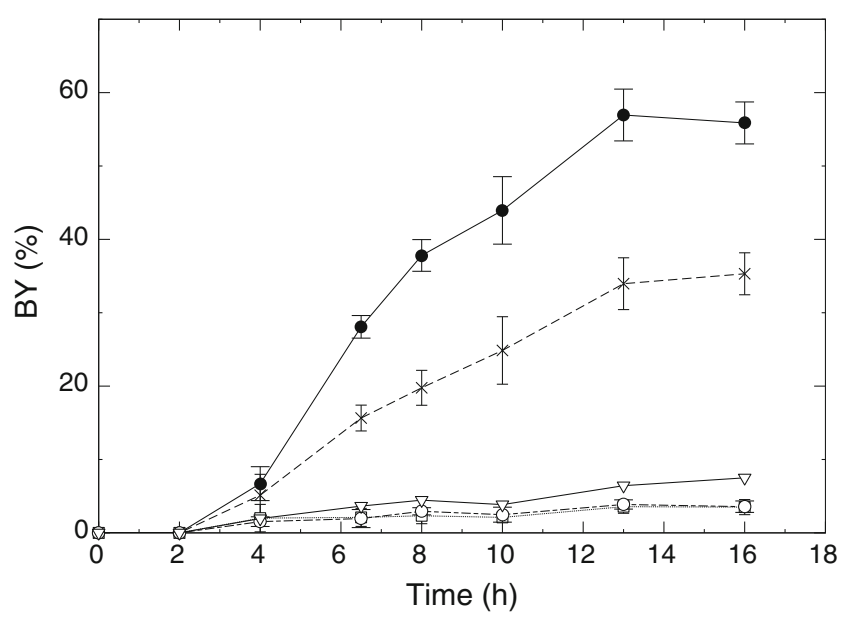

Fig. 5 Curves of BY with the clones; c3 of $n f s B p B A D-N f s B$ $(\longrightarrow)$ and $\mathrm{c} 2$ of $n f s A \mathrm{pBAD}-\mathrm{NfsA}(--\times-)$ and the control strains: wild-type ( $\square-$ - $), n f s A$ mutant $(\square-\square)$ and $n f s B$ mutant $(----)$. Both clones selected were induced with $0.02 \%(w / v)$ of L-arabinose. The samples were taken at $0,2,4,6.5,8,10,13$ and $16 \mathrm{~h}$. The average and SD were calculated of at least three independent replicates 
The production of D-DIBOA in both strains increased linearly from 4 to around $13 \mathrm{~h}$ (Fig. 5) reaching a maximum BY of $35.31 \% \pm 2.47 \%$ for $n f s A$ pBAD-NfsA at $16 \mathrm{~h}$ and $55.87 \% \pm 4.35 \%$ at $13 \mathrm{~h}$ for $n f s B \mathrm{pBAD}-\mathrm{NfsB}$. As expected, the wild-type strain presented a lower BY than the above strains but a higher BY than the $n f s A$ and $n f s B$ single mutants (Fig. 5).

\section{Discussion}

Nitroreductases have been identified and characterised in a variety of bacteria: Gram-negative and Gram-positive; symbionts, pathogens, and free living organsims; heterotrophs and phototrophs; mesophilic and thermophilic species; and aerobic and anaerobic facultative bacteria. In the last few years, these enzymes have received attention due to their environmental, biotechnological and clinical applications (Roldán et al. 2008). These enzymes are thought to participate in oxidative stress response (Liochev et al. 1994, 1999), although their physiological role has not been elucidated yet (Umezawa et al. 2008). Nitroreductases could also be involved in the reduction of non-natural substrates as xenobiotic compounds released by humans activities in the recent years (Rau and Stolz 2003).

In a previous work, we have demonstrated that $E$. coli is able to biotransform the precursor [4-hydroxy- $(2 \mathrm{H})-1,4-$ benzoxazin-3(4H)-one] to D-DIBOA (Valle et al. 2011), a compound with interesting biological properties (Macías et al. 2006a). Here, we studied the possible role of the NfsA and $\mathrm{NfsB}$ nitroreductases and the flavin reductase NemA in this biotransformation. In our approach, we generated the single and double mutants of these genes and then we analysed their BY and SP. However, we will only discuss the BY values because the behaviour of both parameters was very similar in all the strains. This would indicate that the differences in the biotransformation ability were not due to the difference in the biomass concentration (Fig. 3). On the other hand, these enzymes do not seem to be essential for central metabolism because biomass production was not affected in the mutants compared to the wild-type.

According to the BY obtained with all the mutants, only the NfsA and $\mathrm{NfsB}$ nitroreductases were involved in the conversion of the precursor to D-DIBOA in our experimental conditions. It has been reported that these enzymes catalyse similar biotransformations as the reduction of the nitro group of nitrofurazone. In this previous work, the double mutant $n f_{S} A / n f_{S} B$ constructed by Rau and Stolz (2003) presented only a $10 \%$ of activity respect to the $E$. coli wild-type strain activity. Mutants inactivated in the $n f_{s} B$ gene presented higher BY than mutants inactivated in the $n f_{S} A$ gene (Fig. 3), indicating that NfsA was more active toward the precursor than $\mathrm{NfsB}$, in vivo. It has been reported that in the reduction of the nitro group of 4-nitrophenol, tested in vitro, the specific activity of NfsA was 5-fold higher than for NfsB (Zenno et al. 1996a,b). Furthermore, the NfsA activities tested in other nitro compounds, like as nitrofurazone, nitrofurantoin, 4-nitrobenzoate or 4nitrotoluene, were higher than for NfsB. Two reasons could explain these results. Firstly, the NfsB protein seems to be expressed at a lower rate than NfsA (Bryant et al. 1981; Kobori et al. 2001; Zenno et al. 1996a,b). Secondly, the $K_{\mathrm{m}}$ value reported for $\mathrm{NfsB}$ is twice that of $\mathrm{NfsA}$, when chromate is used as the substrate, which would indicate that $\mathrm{NfsA}$ has more affinity than NfsB for this kind of substrates (Kwak et al. 2003). Therefore, the higher yields observed with the $n f_{S} B$ single mutant compared to the $n f_{S} A$ single mutant could be explained by enzyme concentration and/or substrate affinity effects (Fig. 3).

The BY of the $n f_{S A} A$ nemA double mutant was slightly higher than in the $n f_{S} A$ single mutant. This would indicate that there is indirect and unknown interaction between NemA activity and the biotransformation of the precursor by NfsB enzyme (Fig. 3).

In order to confirm that $\mathrm{Nfs} A$ and $\mathrm{NfsB}$ were responsible for the biotransformation of the precursor to D-BIBOA, both ORF were cloned into $\mathrm{pBAD} / \mathrm{His} \mathrm{A}$, a vector that allows different levels of expression of genes under the araBAD promoter by adding different concentrations of Larabinose to the culture medium (Guzman et al. 1995). When the $n f_{s} A$ and $n f_{s} B$ single mutants were complemented with the corresponding genes cloned in $\mathrm{pBAD}$, the loss of activity was recovered at the lowest inducer concentration tested in our experiments $(0.0002 \%$ of L-arabinose). As the inductor concentration was increased, the BY considerably increased up to 9.6 for $n f s B \mathrm{pBAD}-\mathrm{NfsB}$ and 6.5 for $n f s A \mathrm{p}$ BAD-NfsA (Fig. 4). These yields are close to the values obtained in the chemical process $(77 \%)$ described in Macías et al. (2006b).

Regarding the question about which of these two genes $\left(n f_{S A}\right.$ or $n f_{S} B$ ) has a more active role in the biotransformation of the precursor, the results obtained from the gene inactivation or the overexpression experiments are somehow contradictory. On the one hand, mutagenesis experiments suggested that NfsA is the main contributor to the biotransformation, but on the other hand, when $\mathrm{NfsB}$ protein was overexpressed in the $n f_{s} B$ mutant ( $n f_{s} B \mathrm{pBAD}-\mathrm{NfsB}$ strain), the BY was higher than when NfsA was overexpressed in the $n f s A$ mutant ( $n f s B$ pBAD-NfsB strain). These experiments would reveal the presence of unknown limiting factor(s), being probably one of them the availability of the reduced cofactors NADH or/and NADPH within the cells. Whereas the NfsB may use either NADH or NADPH, NfsA can only use NADPH. The only use of NADPH and the lower concentration of this cofactor compared with the one of NADH (Rau and Stolz 2003) could represent a limiting 
factor for NfsA. On the other hand, although NfsB could have lower activity levels than NfsA in normal physiological conditions, when the enzymes are overexpressed, the use of both NADH and NADPH by NfsB may represent an advantage that would explain the higher biotransformation activity observed in the overexpression of NfsB compared to NfsA. This hypothesis would be also supported by the data obtained from the experiments in which NfsA or NfsB were overexpressed in the wild-type strain (wtpBAD-NfsA and wtpBAD-NfsA strains, respectively). In these strains, there is an extra copy of $n f s A$ (Fig. 4a) or $n f s B$ (Fig. 4b) genes provided by the endogenous $n f_{S} A$ and/or $n f_{S} B$ genes. However, in both cases, the presence of an extra copy does not reveal an increment of the biotransformation ability respect to the recovery of the biotransformation capacity experiments with the $n f s A \mathrm{pBAD}-\mathrm{Nfs} \mathrm{A}$ and nfsBpBAD$\mathrm{NfsB}$ strains. These experiments would suggest that $\mathrm{NfsA}$ and NfsB concentration within the cells are not limiting the biotransformation in the overexpression experiments. In these cases, probably other factors like the redox cofactors availability are limiting the process. The importance of redox cofactors as limiting factor for these enzymes have been previously reported in an in vitro assay in which the reduction of TNT by $E$. coli nitroreductases significantly increased when redox cofactors were added to the reaction (Rau and Stolz 2003). In conclusion, taking together, the mutagenesis and overexpression experiments would reveal a higher efficiency of NfsA respect to NfsB but less versatility in the usage of redox cofactors in the synthesis of $\mathrm{D}$ DIBOA from the precursor.

In summary, our results demonstrate the implication of the NfsA and NfsB nitroreductases in the biotransformation of the precursor to D-DIBOA by E. coli. Furthermore, we show that the genetic improvement of these bacteria can represent a plausible alternative to the second step of the chemical synthesis of D-BIBOA, which is a complex and expensive process. The aim of our future efforts will be to achieve an increase of the production yield of D-DIBOA by the optimisation of culture medium and the usage of several E. coli strains.

Acknowledgements This work was supported by the Consejería de Innovación, Ciencia y Empresa de la Junta de Andalucía through the Project for Excellence P06-01399 (2006) and the PAIDI group CTS569; and the Co-operation Office of European Union in the LFA program called Bioprocess: Clean Technologies in the protection and sustainability of the environment with contract no.: AML/190901/06/ 18414/II-0548-FC-FA. The authors wish to thank PhD J.L. Ramos of the Consejo Superior de Investigaciones Científicas (CSIC) who provided the E. coli strain AB502NemA and Guillermo Gosset from the Instituto de Biotecnología from the Universidad Nacional Autónoma de Mexico (UNAM) for providing the detailed protocol for gene inactivation. Jorge Yañéz is acknowledged by primers synthesis.

\section{References}

Blattner FR, Plunkett G, Bloch CA, Perna NT, Burland V, Riley M, ColladoVides J, Glasner JD, Rode CK, Mayhew GF, Gregor J, Davis NW, Kirkpatrick HA, Goeden MA, Rose DJ, Mau B, Shao Y (1997) The complete genome sequence of Escherichia coli K-12. Science 277:1453-1462

Bryant DW, Mccalla DR, Leeksma M, Laneuville P (1981) Type-I nitroreductases of Escherichia coli. Can J Microbiol 27:81-86

Datsenko KA, Wanner BL (2000) One-step inactivation of chromosomal genes in Escherichia coli K-12 using PCR products. Proc Natl Acad Sci USA 97:6640-6645

Duke S (1986) Naturally occurring chemical compounds as herbicides. Rev Weed Sci 2:5-44

Esteve-Nuñez A, Caballero A, Ramos JL (2001) Biological degradation of 2,4,6-trinitrotoluene. Microbiol Mol Biol Rev 65:335-352

French CE, Nicklin S, Bruce NC (1998) Aerobic degradation of 2,4,6trinitrotoluene by Enterobacter cloacae PB2 and by pentaerythritol tetranitrate reductase. Appl Environ Microbiol 64:2864-2868

González-Pérez MM, van Pieter D, Rolf MW, Ramos JL (2007) Escherichia coli has multiple enzymes that attack TNT and release nitrogen for growth. Environ Microbiol 9:1535-1540

Greenberg AE, Clesceri LS, Eaton AD (1992) Standard methods for the examination of water and wastewarer, 18th edn. APHA, Washington, 936

Guzman LM, Belin D, Carson MJ, Beckwith J (1995) Tight regulation, modulation, and high-level expression by vectors containing the arabinose $\mathrm{P}_{\mathrm{BAD}}$ promoter. J Bacteriol 177:4121-4130

Hamilton RH, Bandurski RS, Reusch WH (1962) Isolation and characterization of a cyclic hydroxamate from Zea mays. Cereal Chem 39:107-113

Honkanen E, Virtanen AI (1960) Precursors of benzoxazolinone in rye plants, II. Precursor I, the glucoside. Acta Chem Scand 14:504 507

Kobori T, Sasaki H, Lee WC, Zenno S, Saigo K, Murphy MEP, Tanokura M (2001) Structure and site-directed mutagenesis of a flavoprotein from Escherichia coli that reduces nitrocompounds —alteration of pyridine nucleotide binding by a single amino acid substitution. J Biol Chem 276:2816-2823

Kwak YH, Lee DS, Kim HB (2003) Vibrio harveyi nitroreductase is also a chromate reductase. Appl Environ Microbiol 69:4390 4395

Liochev SI, Hausladen A, Beyer WF, Fridovich I (1994) NADPHferredoxin oxidoreductase acts as a paraquat diaphorase and is a member of the SoxRS regulon. Proc Natl Acad Sci USA 91:13281331

Liochev SI, Hausladen A, Fridovich I (1999) Nitroreductase A is regulated as a member of the soxRS regulon of Escherichia coli. Proc Natl Acad Sci USA 96:3537-3539

Macías FA, Molinillo JMG, Galindo JCG, Varela RM, Simonet AM, Castellano D (2001) The use of allelopathic studies in the search for natural herbicides. J Crop Prod 4:237-255

Macías FA, Marín D, Oliveros-Bastidas A, Castellano D, Simonet AM, Molinillo JMG (2006a) Structure-activity relationship (SAR) studies of benzoxazinones, their degradation products, and analogues. Phytotoxicity on problematic weeds Avena fatua L. and Lolium rigidum Gaud. J Agric Food Chem 54:1040-1048

Macías FA, Marín D, Oliveros-Bastidas A, Chinchilla D, Simonet AM, Molinillo JMG (2006b) Isolation and synthesis of allelochemicals from Gramineae: benzoxazinones and related compounds. J Agric Food Chem 54:991-1000

Macías FA, Oliveros-Bastidas A, Marín D, Carrera C, Chinchilla N, Molinillo JMG (2008) Plant biocommunicators: their phytotoxicity, degradation studies and potential use as herbicide models. Phytochem Rev 7:179-194 
Macías FA, Marín D, Oliveros-Bastidas A, Molinillo JMG (2009) Rediscovering the bioactivity and ecological role of 1,4-benzoxazinones. Nat Prod Rep 26:478-489

Michael NP, Brehm JK, Anlezark GM, Minton NP (1994) Physical characterization of the Escherichia coli-B gene encoding nitroreductase and its over-expression in Escherichia coli K12. FEMS Microbiol Lett 124:195-202

Rau J, Stolz A (2003) Oxygen-insensitive nitroreductases NfsA and $\mathrm{NfsB}$ of Escherichia coli function under anaerobic conditions as lawsone-dependent Azo reductases. Appl Environ Microbiol 69:3448-3455

Roldán MD, Pérez-Reinado E, Castillo F, Moreno-Vivián C (2008) Reduction of polynitroaromatic compounds: the bacterial nitroreductases. FEMS Microbiol Rev 32:474-500

Umezawa Y, Shimada T, Kori A, Yamada K, Ishihama A (2008) The uncharacterized transcription factor YdhM is the regulator of the nemA Gene, encoding N-ethylmaleimide reductase. J Bacteriol 190:5890-5897

Valle A, Cabrera G, Molinillo JMG, Gómez JM, Macías FA, Cantero D (2011) Biotransformation of ethyl 2-(2'-nitrophenoxy)acetate to benzohydroxamic acid (D-DIBOA) by Escherichia coli. Process Biochem 46:358-364

Whiteway J, Koziarz P, Veall J, Sandhu N, Kumar P, Hoecher B, Lambert IB (1998) Oxygen-insensitive nitroreductases: analysis of the roles of $n f s A$ and $n f s B$ in development of resistance to 5-nitrofuran derivatives in Escherichia coli. J Bacteriol 180:55295539

Williams RE, Bruce NC (2002) 'New uses for an old enzyme'- the Old Yellow Enzyme family of flavoenzymes. Microbiol 148:1607-1614

Williams RE, Rathbone DA, Scrutton NS, Bruce NC (2004) Biotransformation of explosives by the old yellow enzyme family of flavoproteins. Appl Environ Microbiol 70:3566-3574

Yin H, Wood TK, Smets BF (2005) Reductive transformation of TNT by Escherichia coli: pathway description. Appl Microbiol Biotechnol 67:397-404

Zenno S, Koike H, Kumar AN, Jayaraman R, Tanokura M, Saigo K (1996a) Biochemical characterization of NfsA, the Escherichia coli major nitroreductase exhibiting a high amino acid sequence homology to Frp, a Vibrio harveyi flavin oxidoreductase. J Bacteriol 178:4508-4514

Zenno S, Koike H, Tanokura M, Saigo K (1996b) Gene cloning, purification, and characterization of $\mathrm{NfsB}$, a minor oxygeninsensitive nitroreductase from Escherichia coli, similar in biochemical properties to FRaseI, the major flavin reductase in Vibrio fischeri. J Biochem 120:736-744

Zenno S, Kobori T, Tanokura M, Saigo K (1998) Conversion of NfsA, the major Escherichia coli nitroreductase, to a flavin reductase with an activity similar to that of Frp, a flavin reductase in Vibrio harveyi, by a single amino acid substitution. J Bacteriol 180:422425 